\title{
Optimizing Sleeves Pattern for Vietnamese Airlines Stewardess Uniform--Ao Dai
}

\author{
Rui Zhang ${ }^{1, a}$, Ngo Thi Viet Ha ${ }^{1,2, b}$, Jianping Wang ${ }^{1, c^{*}}$ \\ ${ }^{1}$ Fashion Institute, Donghua University, Shanghai 200051, China \\ ${ }^{2}$ School of Textile-Leather and Fashion, Hanoi University of Science and Technology, Hanoi, \\ Vietnam \\ azhangruiheart@126.com, bngothivietha_85@yahoo.com, \\ cwangjp@dhu.edu.cn
}

Keywords: Ao Dai, Airlines stewardess uniform, Raglan sleeves pattern, Five levels of psychological scale method

\begin{abstract}
Ao Dai is Vietnamese national costume and usually used as airlines stewardess uniform. As uniform, Ao Dai is required to be fitted and beautiful. However, the sleeves of Ao Dai limit stewardesses arms motion when they raise hands over head or move arms frequently. The sleeves of Ao Dai are one-piece raglan sleeves. By changing sleeve width with $-0.5 \mathrm{~cm}, 0 \mathrm{~cm}, 0.5 \mathrm{~cm}, 1 \mathrm{~cm}$ and $1.5 \mathrm{~cm}, 5$ samples were made. 8 subjects wore samples to evaluate the comfort and beauty of Ao Dai sleeves with five levels of psychological scale method. It could be seen that, sample $\mathrm{A}_{3}$ was the most appropriate one, which could satisfy comfort and beauty request. The study identified the suitable sample through optimizing the sleeves pattern which was evaluated with five levels of psychological scale method.
\end{abstract}

\section{Introduction}

As one kind of traditional clothing, Ao Dai known as Vietnamese national costume is very popular [1]. Ao Dai is used as work uniforms in many different fields including Airlines companies [2]. As Airlines stewardess uniform, Ao Dai promotes Vietnamese costume culture with its unique folk style. In order to be fitted and beautiful, the sleeves of Ao Dai are designed as one-piece raglan sleeves with no shoulder seam, small armhole depth. Due to fitted, the sleeves of Ao Dai limit the freedom of arms motion when stewardesses raise hands over head or move arms frequently. Based on the above requirements, optimizing the pattern of Ao Dai sleeves to obtain suitable sleeves with aesthetic and comfort is meaningful.

\section{Methodology}

\section{One-piece raglan sleeve analysis}

One-piece raglan sleeve, namely the front piece sleeve and back piece sleeve are joined to be one piece in sleeve center line with no seam. The sleeve center line slope (usually $0^{\circ} \sim 20^{\circ}$ ) of one-piece sleeve is based on shoulder slope ( average Asian women shoulder slope is $20^{\circ}$ [3] ), and the connection points of sleeve and body pieces are set on front and back armpit points [4]. In general, the bicep line and the sleeve center line slope decrease with the increase of sleeve width.

\section{Vietnamese Ao Dai sleeves structure analysis}

Ao Dai structure draft is formed according to long-time experience, whose style is similar to Chinese cheongsam. Ao Dai sleeves can be classified as raglan sleeves. However they have no visualized angle between sleeve center line and horizontal line. Firstly pieced body structure with sleeve structure together (Fig. 1a), resulting in $23^{\circ}\left(90^{\circ}-67^{\circ}\right)$ between front sleeve center line with horizontal line and $13^{\circ}\left(90^{\circ}-77^{\circ}\right)$ between back sleeve center line with horizontal line. Then 
second rotation, the back angle changed from $13^{\circ}$ to $22^{\circ}$ (Fig. 1b). Through two times of rotation, Ao Dai sleeve center line slope was found to fit well with Asia female' s front $\left(22^{\circ}\right)$ and back $\left(18^{\circ}\right)$ shoulder slope [5].

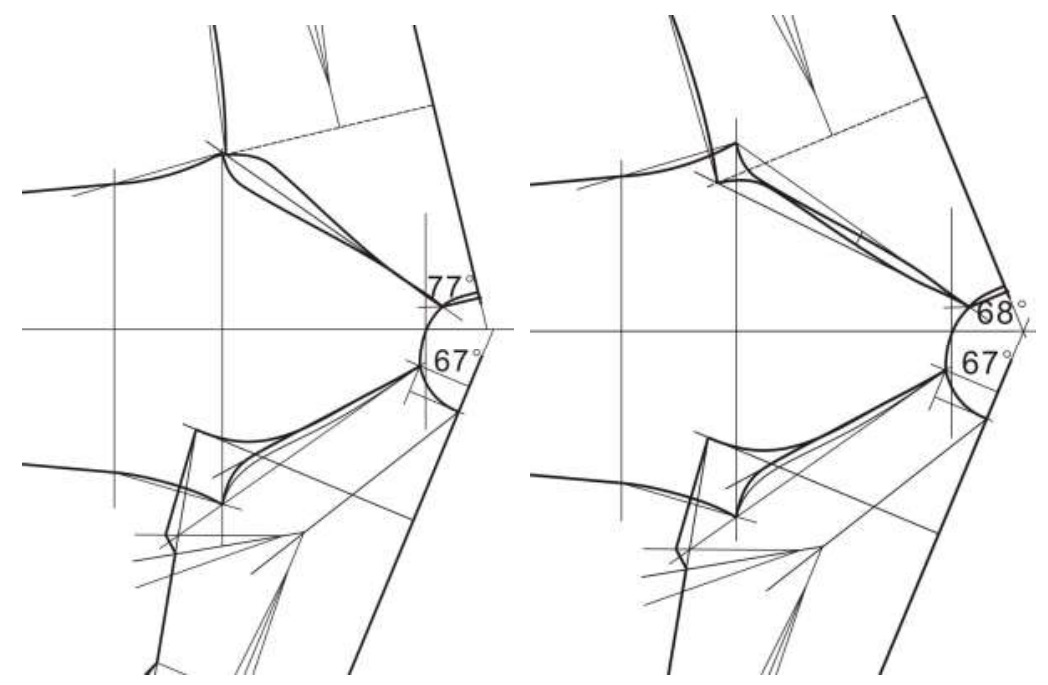

a First Structure analysis b Second structure analysis

Fig. 1: Analysis of Ao Dai body structure and sleeve structure

\section{Ao Dai sleeve structure optimization}

\section{Data}

17 positions data of 40 Human airlines stewardesses were collected (tab. 1) by SPSS to extract the standard figure, whose data were used to optimize Ao Dai sleeves.

\section{Experimental protocol}

Structure was drawn according to the standard figure somatic data. By changing sleeve width with $-0.5 \mathrm{~cm}, 0 \mathrm{~cm}, 0.5 \mathrm{~cm}, 1 \mathrm{~cm}$ and $1.5 \mathrm{~cm}, 5$ samples were made, namely A1, A2, A3, A4 and A5 (Fig. 2) .

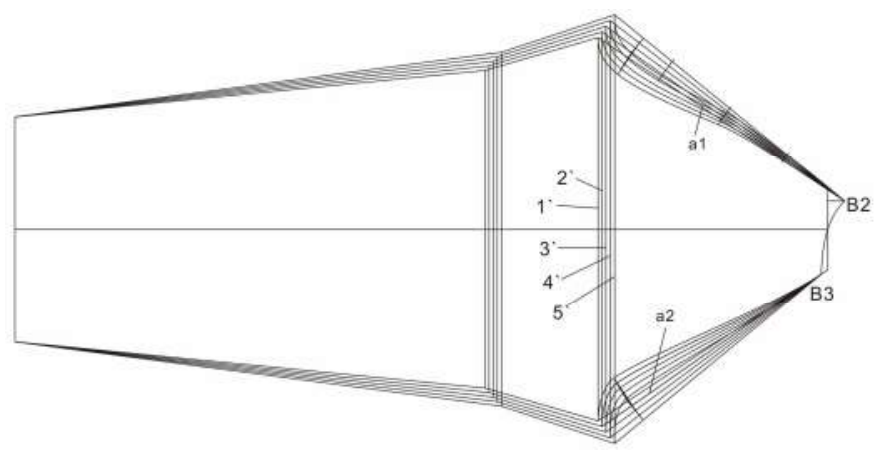

Fig. 2: Optimization of Ao Dai sleeves pattern

5 samples were made by using the same fabric with uniform Ao Dai. 8 subjects of 165/84A were chosen to wear and evaluate aesthetic and comfort of samples subjectively with five levels of psychological scale method. When aesthetic evaluated, subjects should stand naturally and arms droop normally with 5 evaluated positions (shoulder, front armpit, back armpit, upper arm, lower arm). When dynamic comfort evaluated, subjects were required to do 7 representative arm movements for judging the comfort of 6 positions, including above mentioned 5 positions and armhole bottom. The evaluation results were as shown in Fig. 3 Fig. 10. 


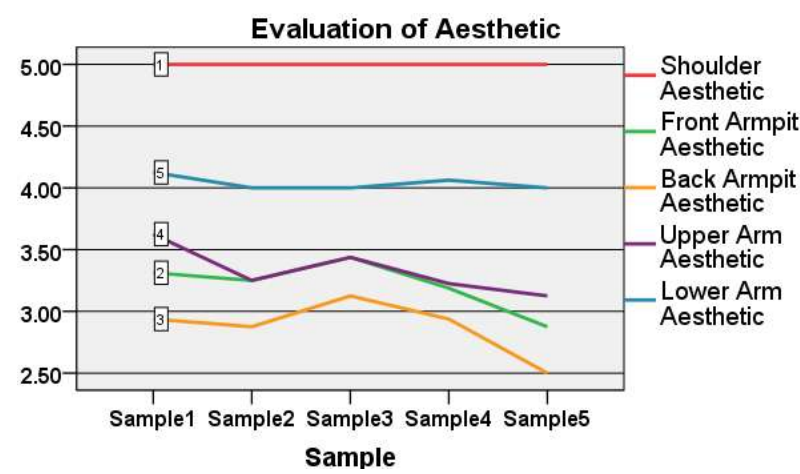

Fig. 3: Evaluation of aesthetic

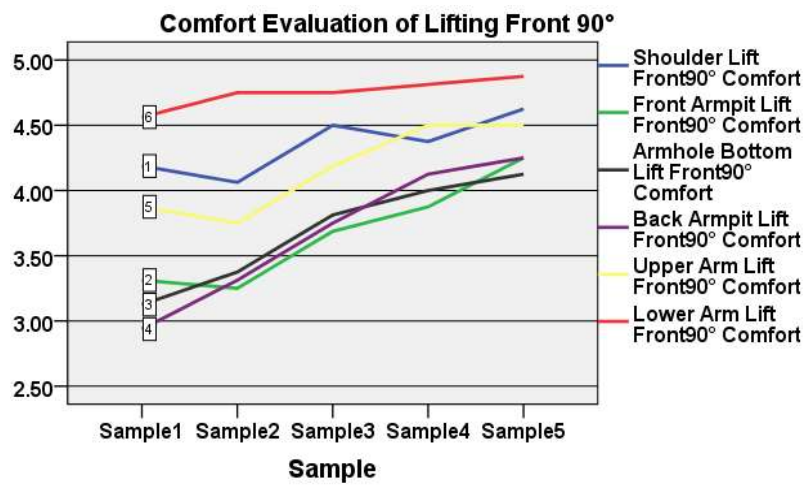

Fig. 5: Comfort evaluation of lifting front $90^{\circ}$

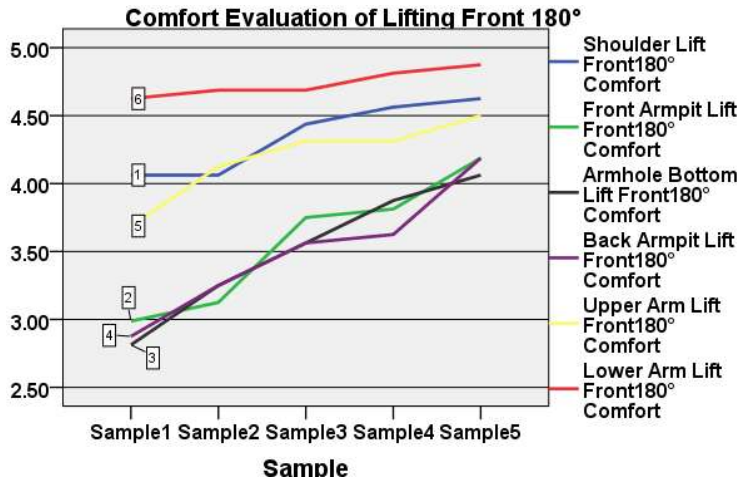

Fig. 7: Comfort evaluation of lifting front $180^{\circ}$

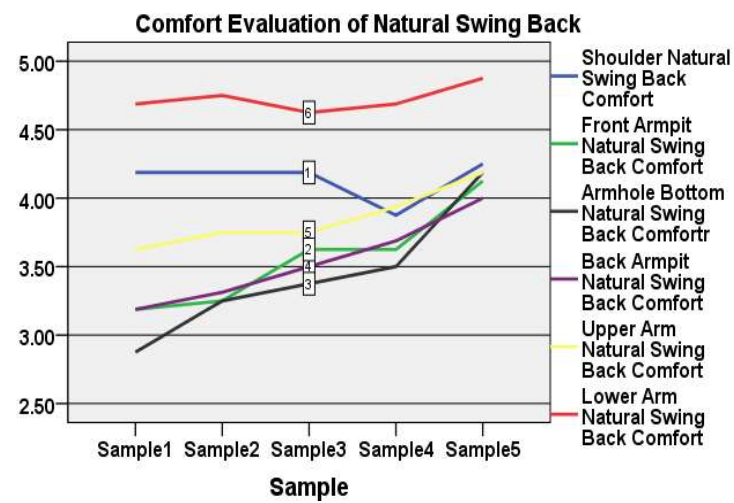

Fig. 9. Comfort evaluation of natural swing back

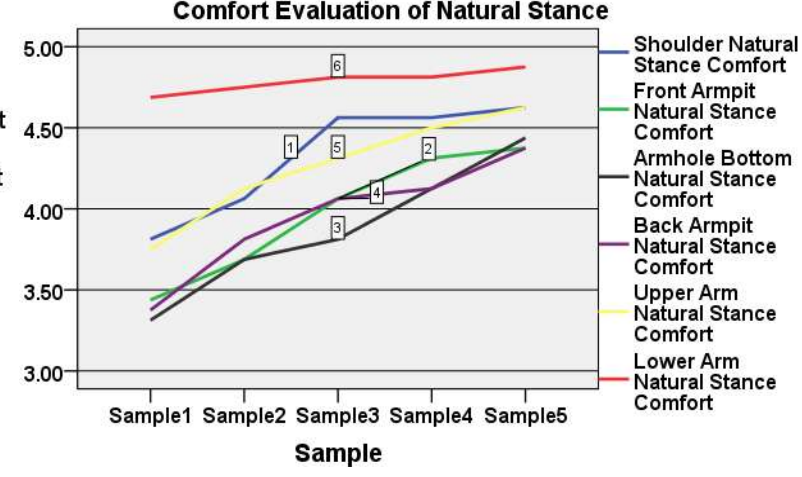

Fig. 4: Comfort evaluation of natural stance

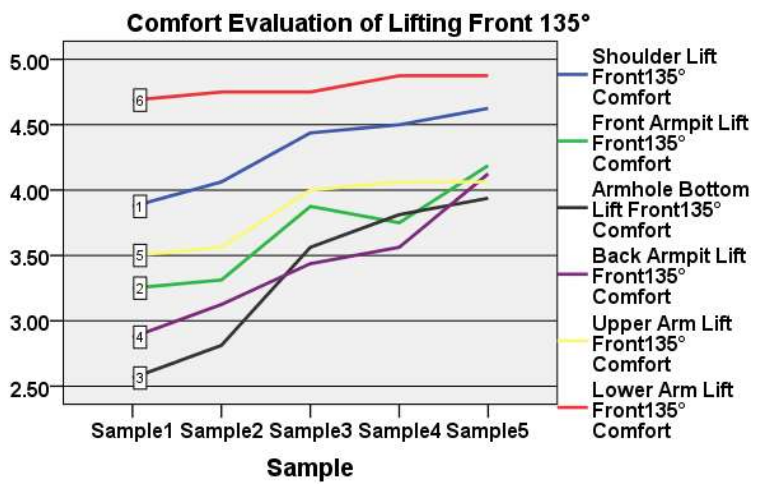

Fig. 6: Comfort evaluation of lifting front $135^{\circ}$

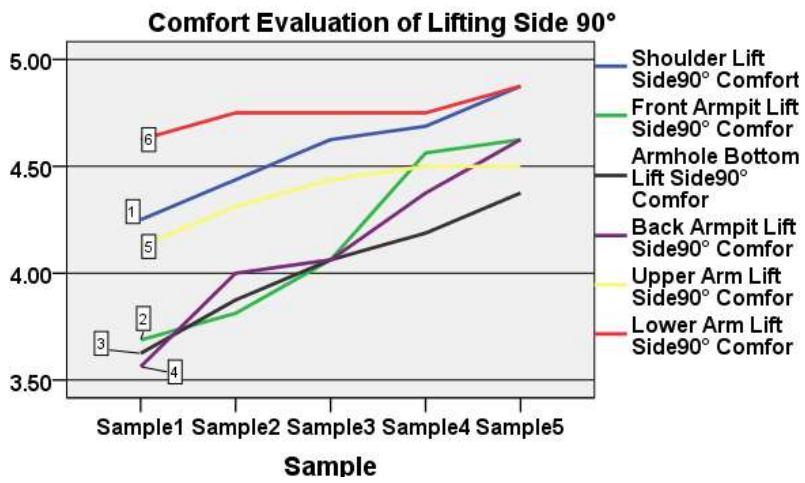

Fig. 8: Comfort evaluation of lifting side $90^{\circ}$

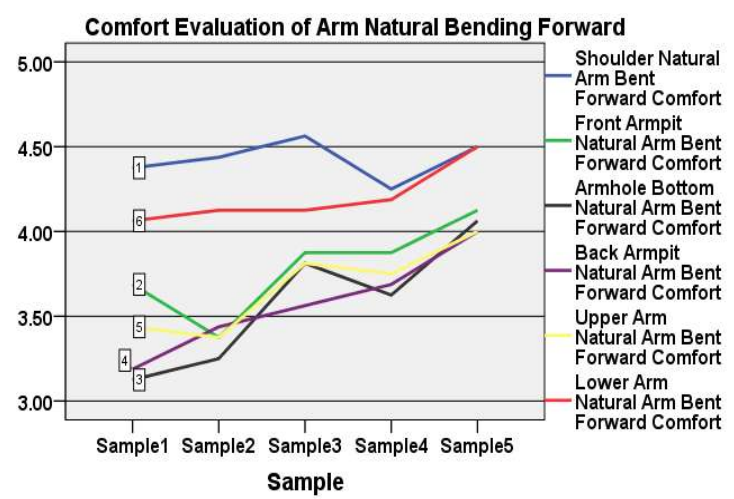

Fig. 10. Comfort evaluation of natural bending forward 
Table 1: Measurement of basic statistics

\begin{tabular}{|c|c|c|c|c|c|c|c|}
\hline & Mean & $\begin{array}{l}\text { Std. Error } \\
\text { of Mean }\end{array}$ & Median & $\begin{array}{c}\text { Std. } \\
\text { Deviation }\end{array}$ & Variance & Minimum & Maximum \\
\hline Height & 53.1825 & .51494 & 54.0000 & 3.25678 & 10.607 & 46.00 & 62.30 \\
\hline Weight & 165.0750 & .50050 & 165.0000 & 3.16542 & 10.020 & 158.00 & 173.50 \\
\hline $\begin{array}{l}\text { Dress } \\
\text { Length }\end{array}$ & 127.6925 & .15285 & 127.9500 & .96673 & .935 & 125.00 & 129.50 \\
\hline FWL & 41.3650 & .08818 & 41.5000 & .55772 & .311 & 40.00 & 42.80 \\
\hline BWL & 38.0400 & .09653 & 38.0000 & .61051 & .373 & 36.00 & 39.00 \\
\hline $\begin{array}{l}\text { BNP to } \\
\text { WP }\end{array}$ & 74.3950 & .33040 & 75.0000 & 2.08965 & 4.367 & 70.00 & 77.50 \\
\hline S.L. & 55.4925 & .23652 & 56.0000 & 1.49587 & 2.238 & 52.50 & 58.00 \\
\hline N.L. & 36.0300 & .10973 & 36.0000 & .69400 & .482 & 34.00 & 37.50 \\
\hline B & 84.2450 & .37788 & 83.8000 & 2.38993 & 5.712 & 81.00 & 92.00 \\
\hline W & 67.8925 & .52448 & 68.0000 & 3.31712 & 11.003 & 60.00 & 74.50 \\
\hline $\mathrm{H}$ & 90.1000 & .44979 & 90.0000 & 2.84470 & 8.092 & 84.00 & 96.00 \\
\hline A.G. & 33.9650 & .12893 & 34.1000 & .81541 & .665 & 31.00 & 35.20 \\
\hline B.C. & 25.9500 & .18077 & 26.2000 & 1.14332 & 1.307 & 22.00 & 27.30 \\
\hline P.S. & 19.6025 & .22585 & 19.3000 & 1.42837 & 2.040 & 17.80 & 22.00 \\
\hline $\begin{array}{l}\text { FNP to } \\
\text { Bust }\end{array}$ & 19.0675 & .10606 & 19.0000 & .67078 & .450 & 18.00 & 21.00 \\
\hline N.B. & 16.6475 & .09641 & 16.5000 & .60974 & .372 & 15.50 & 18.50 \\
\hline S.S. & 21.1950 & .16878 & 21.0000 & 1.06746 & 1.139 & 19.50 & 23.50 \\
\hline
\end{tabular}

\section{Conclusions}

By using five level psychological scale method to evaluate the static beauty and dynamic comfort of the samples, it could be seen that sample A1 was the best on aesthetic, but the worst on comfort; Sample A2 was slightly inferior to the sample A3 on aesthetic, but comfort was poorer; Sample A3 was slightly inferior to the sample A1 on aesthetic, and better on comfort, which could satisfy the working requirements; The sleeves of sample A4 and A5 were loose, so comfort was good, but poor on aesthetic. The aesthetic ranking was $\mathrm{A} 1>\mathrm{A} 3>\mathrm{A} 2>\mathrm{A} 4>\mathrm{A} 5$, and the comfort ranking was A $5>$ A $4>$ A $3>$ A $2>$ A 1 .

Above all, sample A3 could meet the requirements of the stewardess uniform both on aesthetic and comfort.

\section{References}

[1] Bi-guo Luo, The cultural charm of the national costume of Vietnam, J. Nanning College for Vocational Technology, 14 (5) (2009) 20-23.

[2] Le Thi Dung, Research on reform Vietnamese Traditional Garment for High School Girls Ao Dai Uniform Design, D. Donghua University, 2014.

[3] Rui-pu Liu, Design principle and application of apparel pattern, Chinese Textile Press, Beijing, 2008.

[4] Ying Huang. Investigation of connection point between body piece and sleeve of one-piece and two-piece raglan sleeve, J. Journal of Zhejiang Textile and Fashion College, 4 (2011) 21.

[5] Wen-bin Zhang, Clothing structure design, Chinese Textile Press, Beijing, 2006. 